\title{
Modified models about security check in airports
}

\author{
Suting Gao
}

School of North China Electric Power University, Baoding 071000, China

377616544@qq.com

\section{Abstract}

In recent years, the rapid development of air transport industry has brought tremendous benefits to airports. But at the same time, complicated passenger flow also brings new challenges for airports. The greatly increased airport traffic leads to passengers' long waiting time as well as a drop in the quality of service.

In consideration of the above-mentioned situations, we propose two models. In our model we obtain passenger flow volume. We analyze the document check and the security check area. When a passenger needs to spend considerable time passing the document check or the security check area, the following passengers will suffer longer waiting time. By using document check area optimization model, we improve the way of queuing. Our model can obtain the reasonable amount of equipment for different situations, which can ensure security-checking efficiency, quality of service and reducing security costs effectively. So, our models have practical significance for operations at the airport.

Key words: security check, waiting time, investment costs and economic efficiency

\section{Introduction}

We are required to build mathematical models to analyze the related factors between airport operations and passengers' flying experience, and we are required to obtain reasonable measures to satisfy security, short time and high yields simultaneously.

We have three tasks

(1) To determine existing problems in the process.

(2) To modify the current process in several places for greater throughput and minor differences in passengers 'waiting time.

(3) To take cultural differences into account and modify the security system to accommodate these differences.

\section{Improved queuing model}

We can get the number of passengers entering into the zone A per second, namely, we can get the number of passengers entering into the airport per second. We can also get the number of passengers passed zone A per second ${ }^{[1]}$. By comparing, the number of passengers passed zone $\mathrm{A}$ is greater than the number of passengers entering into zone A. So passengers can pass document check smoothly under normal circumstances ${ }^{[2]}$. But there is a proportion of passengers spending a lot of time in zone A. When a passenger needs to take considerable time to check document, passengers after him or her need to queue longer inevitably, which is the problem of zone $A^{[3]}$. In the same way, we can obtain the problem of zone B.

We modify the process of document check and security check to meet the requirements. The modification can shorten the whole waiting time and reduce the variance. 


\subsubsection{Document check area optimization model}

The number of passengers entering the airport per second can be obtained according to TSA Pre-Check Arrival Times and Regular Pax Arrival Times ${ }^{[4]}$.

The time a passenger would spend checking document can be obtained according to ID Check Process Time 1 and ID Check Process Time 2. Then the number of passengers passed zone A per second can be obtained.

We simulate document checks based on un-improved process ${ }^{[5]} .100$ regular passengers enter zone A, with one passenger spending 500 seconds checking in, every passenger's waiting time is shown in Figure 1.

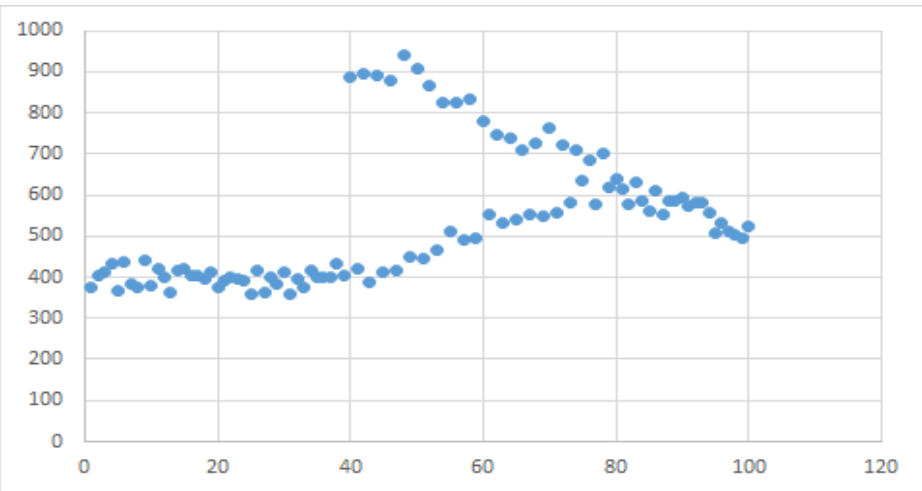

Figure 1 Passengers' waiting time in un-improved zone A

Because this kind of situation is recurrent, we modify zone A to shorten the whole waiting time and reduce differences of waiting time between passengers.

We adopt document check area optimization model in zone A, which is shown in Figure ${ }^{[6][7]}$.

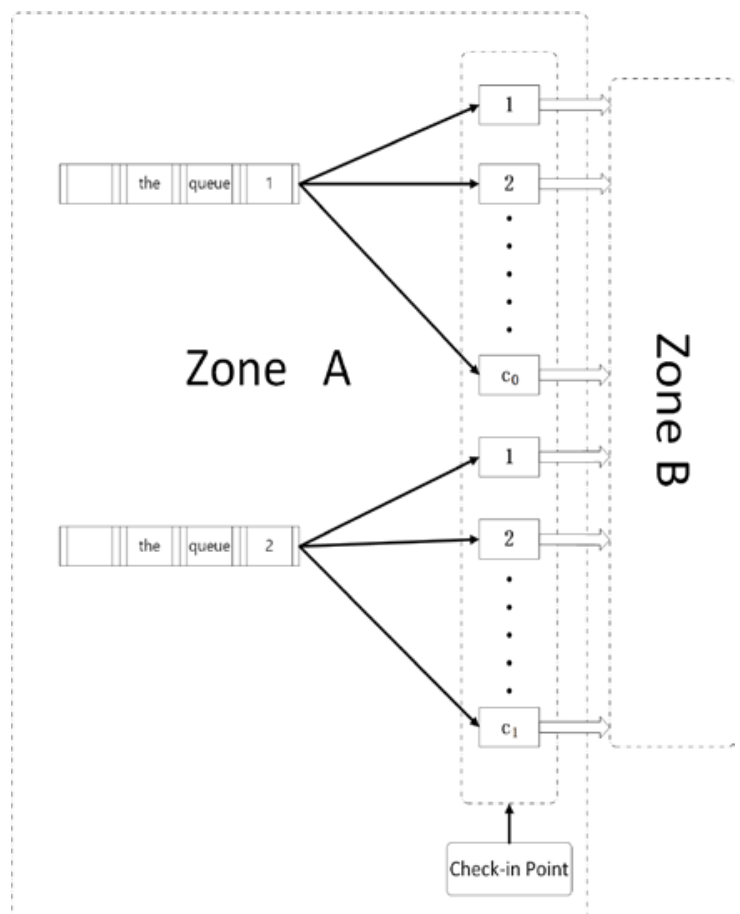

Figure 2 improved zone A

We simulate document checks based on improved process, 100 regular passengers enter zone A and queue up, with one passenger spending 500 seconds 
checking in, every passenger's waiting time is shown in Figure 3.

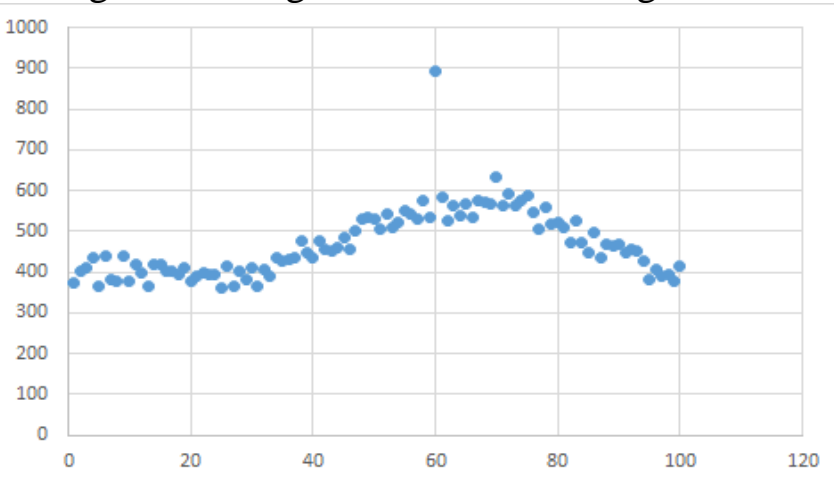

Figure 3 Passengers'waiting time in improved zone A

The improved process would shorten the whole waiting time, at the same time, variance of waiting time between passengers would be reduced. The following Table 1shows the result:

Table 1. The waiting time before and after optimizing

\begin{tabular}{c|c|c}
\hline Waiting time & Before optimizing & After optimizing \\
\hline Average & 536.09 & 468.59 \\
\hline Variance & 24270.38 & 6853.422 \\
\hline
\end{tabular}

The average value of passengers' waiting time decreases by $12.5912 \%$

The variance in waiting time reduces by $71.7622 \%$

Improved zone A can avoid influencing other passengers to get serviced normally when there is a passenger needs to spend longer time checking document. So, the airport can make sure of first-come-first-served basis.

Improved zone A can shorten average waiting time. We can explain our improved zone A can improve passenger throughput and reduce variance in waiting time reasonably.

\section{Conclusions}

In our model we obtain passenger flow volume. We analyze the document check and the security check area. When a passenger needs to spend considerable time passing the document check or the security check area, the following passengers will suffer longer waiting time. By using document check area optimization model, we improve the way of queuing.

\section{Strengths}

- According to the difference of rush hours and non-rush hours, we think that passenger flow meet different $\lambda$ Poisson distribution by stages .This way can increase the authenticity of our models.

- We use Queuing model and Hierarchical and Sorting Queuing model, which reduce passengers' average waiting time. We simulate the models for many times to validate our correctness.

- In allusion to rush hours and non-rush hours, we create number of service counters database by using Dynamic Service Desks model .This make our model dynamic. 
- As the saying goes, the customer is always right .When we set the weight of objective function, we give the customer waiting time cost is larger. And we think this behavior will make the airport TSA have long-term benefit.

\section{Weaknesses}

- The assumption passenger flow meet different $\lambda$ Poisson distribution by stages has good authenticity. However, the actual situation is more complicated.

- For difference of rush hours and non-rush hours, we just give rough $\lambda$, in statistical sense. But we don't looking for a specific airport flight data to make predictions

\section{Future work}

If the airport passenger traffic is a more complicated process ${ }^{[8]}$, it will make our model more reasonable. Therefore, we will get further research base on different flight situation.

\section{Reference}

[1] Aviation Baggage Screening Strategies: To Screen or Not to Screen, that is the question [D], 2003.

[2] GU Yang, Zheng Min, Zhou Hang, Li Yue. Research on Dynamic Allocations of Airport Security Check Resources [J]. Aeronautical Computing Technique, 2016, 46(5)

[3] Zeng Junjie. Settings and Optimization of Airport Security Check [J], Knowledge Economy, 2009(6X):173-174.

[4] Gao Jinhua, Li Jie. Application of Erlang Queuing Model in Airport Passenger Terminals[J]. Journal of Civil Aviation University of China, 2007, 25(2):48-51.

[5] Xiao Yaohua, Deng Bo, Huo Haochao, Ye Shiqi, Hu Daiqiang. Optimal Screening Strategy for Aviation Security[J]. Journal of Jinan University (Natural Science \& Medicine Edition), 2003, 24(5):35-42.

[6] Si Shoukui, Sun Yaoliang, Sun Xiqing. Mathematical modeling algorithm and application[M]. National Defense Industry Press, 2015

[7] Sun Yun. Research on Airport Decision Support[D]. Nanjing University of Aeronautics and Astronautics, 2008

[8]Ji Yun, Li Houpeng, Fu Xinyu. Analysis of Airport Passenger Flow Quantity Based on Compound Poisson Process[J]. Journal of Chongqing Technology and Business University(Natural Science Edition), 2013, 30(5):9-11. 